\title{
Revisão de Parentheca Berg (Heteroptera, Pentatomidae, Pentatomini) ${ }^{1}$
}

\author{
Luiz Alexandre Campos ${ }^{2}$ \\ Jocélia Grazia ${ }^{2,3}$
}

\begin{abstract}
Review of Parentheca Berg (Heteroptera, Pentatomidae, Pentatomini). The neotropical genus Parentheca Berg, 1891 is revised and redescribed with emphasis on male and female genitalia, and placed into "section 1" of Pentatomini which includes those genera lacking a basal spine or tubercle at the abdominal venter. Parentheca subfurcata Berg, 1891 (type species) and Parentheca aeliomorpha Berg, 1891, both from Argentina are redescribed, also with emphasis on male and female genitalia, which are fully illustrated. The lectotype and paralectotype of $P$. aeliomorpha are designated.

KEY WORDS. Heteroptera, Pentatomini, Parentheca, taxonomy, morphology of genitalia
\end{abstract}

O gênero Parentheca foi descrito por BERG (1891) para incluir duas novas espécies, a espécie-tipo por designação subseqüente Parentheca subfurcata (KIRKALDY 1909: 90) (de Córdoba, Argentina) e Parentheca aeliomorpha (de Misiones, Argentina e do Paraguai). Ambas foram citadas em catálogo por KIRKALDY (1909: 90-91). RUFFINELLI \& PIRÁN (1959: 18) citaram P. aeliomorpha para o Uruguai e PIRÁN (1970: 127) para o Brasil (Rio Grande do Sul), ampliando a distribuição do gênero e da espécie.

Parentheca, por não apresentar um espinho ou tubérculo na base ventral do abdome, situa-se dentro da "seção 1" de Pentatomini proposta por ROLSTON \& MCDONALD (1984).

A dissecção, o tratamento das genitálias e a confecção das ilustrações seguem CAMPOS \& GRAZIA (1995). A terminologia das peças da genitália está de acordo com Dupuis $(1955,1970)$.

Todas as medidas estão expressas em milímetros e correspondem à média e valores extremos entre parênteses. Para $P$. subfurcata macho as medidas correspondem aos valores absolutos do único exemplar disponível.

As siglas das coleções do material estudado são citadas a seguir. Agradecemos aos curadores e/ou responsáveis pelo empréstimo do material cujos nomes são citados entre parênteses: (CPJB) Coleção particular de J. Becker, Rio de Janeiro, Rio de Janeiro, Brasil; (DZUP) Departamento de Zoologia, Universidade Federal do Paraná, Curitiba, Paraná, Brasil (K. Zanol); (MACN) Museo Argentino de

1) Realizado com auxilio da FAPERGS, processo $95 / 60103.0$

2) Departamento de Zoologia, Universidade Federal do Rio Grande do Sul. Avenida Paulo Gama, 90046-900 Porto Alegre, Rio Grande do Sul, Brasil.

3) Bolsista do CNPq. E-mail: jocelia@vortex.ufrgs.br 
Ciencias Naturales "Bernardino Rivadavia", Buenos Aires, Argentina (A. Bachmann); (MZSP) Museu de Zoologia, Universidade de São Paulo, São Paulo, São Paulo, Brasil (U.R. Martins); (UFRG) Departamento de Zoologia, Universidade Federal do Rio Grande do Sul, Porto Alegre, Rio Grande do Sul, Brasil (J. Grazia).

\section{Parentheca Berg, 1891}

Figs 1-20

Parentheca Berg, 1891: 280-282. - Kirkaldy, 1909: 90-91. - Ruffinelli \& Pirán, 1959: 18. - Pirán, 1970: 127.

Espécie-tipo: $P$. subfurcata Berg, 1891, por designação subseqüente.

Diagnose. Corpo de contorno oval, maior largura ao nível do terceiro segmento abdominal, coloração ocre a cúprea e pontuações cobre a marrom escuras; superfície dorsal plana, ventral convexa. Cabeça mais longa que larga; jugas mais longas que o clípeo e afastadas no ápice. Olhos pequenos, diâmetro de um olho equivalente a menos de 1/6 da largura da cabeça. Ocelos diminutos, quase imperceptíveis, diâmetro de um ocelo equivalente a cerca de duas vezes o diâmetro de uma das pontuações da cabeça. Rostro curto, apenas atingindo a base do abdome. Cicatrizes do pronoto conspícuas. Peritrema ostiolar auricular, reduzido, com maior diâmetro equivalente a metade do maior diâmetro ocular. Abdome ventralmente sem espinho ou tubérculo mediano basal.

Redescrição, complementando BERG (1891). Cabeça mais longa que larga; olhos pequenos, de diâmetro equivalente a menos de 1/6 da maior largura da cabeça. Pontuações pequenas, densa e regularmente distribuídas, de coloração cobre a marrom escura. Jugas mais longas que o clípeo, afastadas no ápice. Tubérculos anteníferos encobertos pelas jugas em vista dorsal. Antenas com cinco segmentos, o primeiro não ultrapassando as jugas, com cerca da metade do comprimento do segundo, este ligeiramente mais longo que o terceiro; terceiro e quarto subiguais; quinto tão longo quanto o segundo. Búculas quase atingindo a base da cabeça, com o ápice e a base arredondados, altura uniforme em quase toda a sua extensão; canal entre as búculas estreitado na região anterior ao primeiro segmento do rostro. Primeiro artículo do rostro contido entre as búculas, segundo bem mais longo que o terceiro e quarto artículos reunidos, alcançando as mesocoxas; ápice do rostro atingindo a base do abdome. Pronoto com pontuações cúpreas maiores que as da cabeça, distribuídas mais densamente junto às margens ântero-laterais, menos densamente no disco; linha mediana longitudinal destituída de pontuações; largura umeral cerca de duas vezes e meia o comprimento do pronoto; superfície dorsal uniformemente plana, cicatrizes nitidamente delineadas, não contrastantes com a cor do pronoto, com pontuações no disco; dentículos ântero-laterais obsoletos; ângulos umerais não desenvolvidos. Escutelo com pontuações de tamanho e cor semelhantes às do pronoto, densa e uniformemente distribuídas exceto ao longo da linha longitudinal mediana, onde a pontuação é esparsa; largura basal pouco menor que o comprimento; contorno subtriangular, margens laterais sub-retilíneas, constrição ao nível do freno pouco evidente; ângulos basais com reduzidas manchas negras; ápice arredondado, ultrapassando a base do sexto urotergito. Hemiélitros 


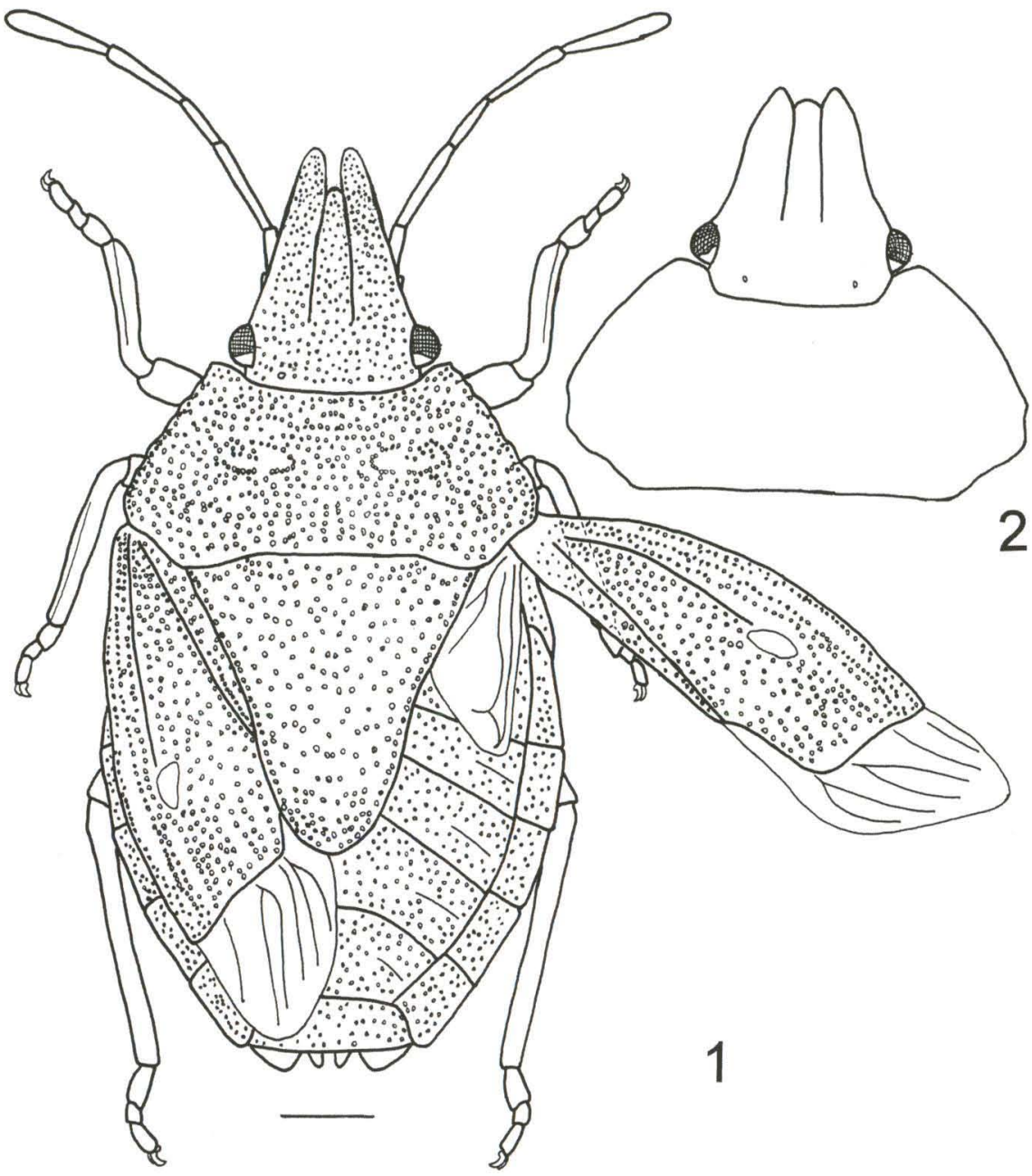

Figs 1-2. (1) Parentheca aeliomorpha fêmea, aspecto dorsal, o hemiélitro direito foi deslocado para expor a reduzida asa posterior; (2) P. subfurcata fêmea, cabeça e pronoto, dorsal. Escala $=1 \mathrm{~mm}$.

com pontuações como no escutelo, porém irregularmente distribuídas; ápice do cório alcançando o nível do sexto segmento do conexivo; mancha no ápice da veia radial presente, não contrastante com a cor de fundo; membranas mais ou menos reduzidas, apresentando cinco a seis veias, raramente bifurcadas. Asas posteriores reduzidas, mal ultrapassando o terceiro urotergito. Prosterno suavemente sulcado. Mesosterno plano e percorrido por uma carena mediana baixa nos dois terços anteriores; levemente escavado no terço posterior. Metasterno suavemente sulcado, com cristas laterais pouco evidentes. Todas as placas esternais recobertas de suave pilosidade. Área evaporatória ocupando a metade interna do mesoepimero, a metade 
interna do metaepisterno, estendendo-se sobre a fenda entre meso e metapleura, não alcançando as margens laterais dos segmentos. Peritrema ostiolar auricular, reduzido, maior diâmetro equivalendo a metade do maior diâmetro ocular. Tíbias dorsalmente sulcadas; pilosas. Conexivo com pontuações menores e mais densas que no restante do corpo; ângulos posteriores arredondados, não pronunciados. Abdome ventralmente convexo, desprovido de espinho ou tubérculo na base do terceiro segmento. Espiráculos abdominais reduzidos. Tricobótrios em pares, localizados atrás e na mesma linha dos espiráculos.

Genitália macho. Pigóforo com os ângulos póstero-laterais pouco pronunciados. Bordo dorsal (Figs 4, 5, 13, 14, bd) com 1+1 ligeiras pregas laterais e avançado na porção mediana de encontro à base do segmento X (Figs 4, 5, 13; 14, bd). Bordo ventral (Figs 3, 12, bv) suavemente côncavo e, na sua porção mais dorsal, com $1+1$ pequenas projeções de cada lado do segmento X (Figs 4, 5, 13, 14, pbv, X). Abertura da taça genital reduzida. Processos do diafragma em forma de carena, originando-se junto ao bordo dorsal e estendendo-se em direção aos ângulos póstero-laterais; carena mais elevada atrás dos parâmeros; externamente à carena a textura do diafragma é bastante irregular, com sulcos longitudinais. Parâmeros (Figs 4, 5, 13, 14, pa) com base subcilíndrica, cabeça subtriangular, ápices recurvados em direção à lateral externa; superficie lateral externa com textura irregular formada por ranhuras transversais (Figs 6,15). Segmento X (proctiger) quadrangular. Phallus: placa basal ampla (Figs 7, 8, 16, 17, pb), mais larga que a phallotheca, com fortes e longos conetivos ventrais (Figs 7, 8, 16, 17, cv). Membramblase muito ampla, trapezoidal (Figs 7, 8, 16, 17, m). Conetivos dorsais longos (Figs 7, 8, 16, 17, cd), quase atingindo o ápice da phallotheca. Phallotheca alongada, subcilíndrica, com abertura posterior, superfície ventral fortemente convexa (Figs 7-9, 16-18, ph). Conjuntiva bem desenvolvida, envolvendo a vesica na base e a encobrindo parcialmente em vista lateral (Figs 7-9, 16-18, cj), com três processos: 1+1 processos ventrais, digitiformes, pouco flexíveis, divergentes a partir da base e arqueados em direção dorsal (Figs 7-9, 16-18, pcj1); um segundo processo de base única e bifurcado em dois prolongamentos digitiformes de ápice agudo que atingem o nível da curvatura dos processos ventrais (Figs 9, 18, pcj2) encoberto, em vista lateral, pela conjuntiva e ventralmente pelos processos digitiformes; o terceiro processo da conjuntiva é único, apical, em forma de tromba, membranoso e bastante flexível, partindo ventralmente entre os processos digitiformes e curvado dorsalmente (Figs $7-9,16-18$, pcj3) (quando não evertido esse processo repousa no espaço existente entre as calotas da vesica). Vesica com 1+1 amplas calotas (Figs 8, 9, 17, 18, v), tendo na base o ductus seminis distalis curto (Figs 9, 18, ds).

Genitália fêmea. Gonocoxitos 8 (Figs 10, 19, gc8) tão longos quanto largos, margem posterior suavemente convexa, bordos suturais justapostos na metade distal, sobrepostos na metade basal. Laterotergitos 8 (Figs 10, 19, la8) mais longos que largos, com margem posterior de contorno triangular. Gonocoxitos 9 (pseudoesternito) (Figs 10, 19, gc9) trapezoidal; braços curtos, atingindo o nível da base dos laterotergitos 9. Laterotergitos 9 (Figs 10, 19, la9) afilados em direção ao ápice arredondado, ultrapassando a banda que une os laterotergitos 8 e quase igualando em comprimento os laterotergitos 8. Gonapófises 9 (Figs 11, 20, g9) com espessa- 


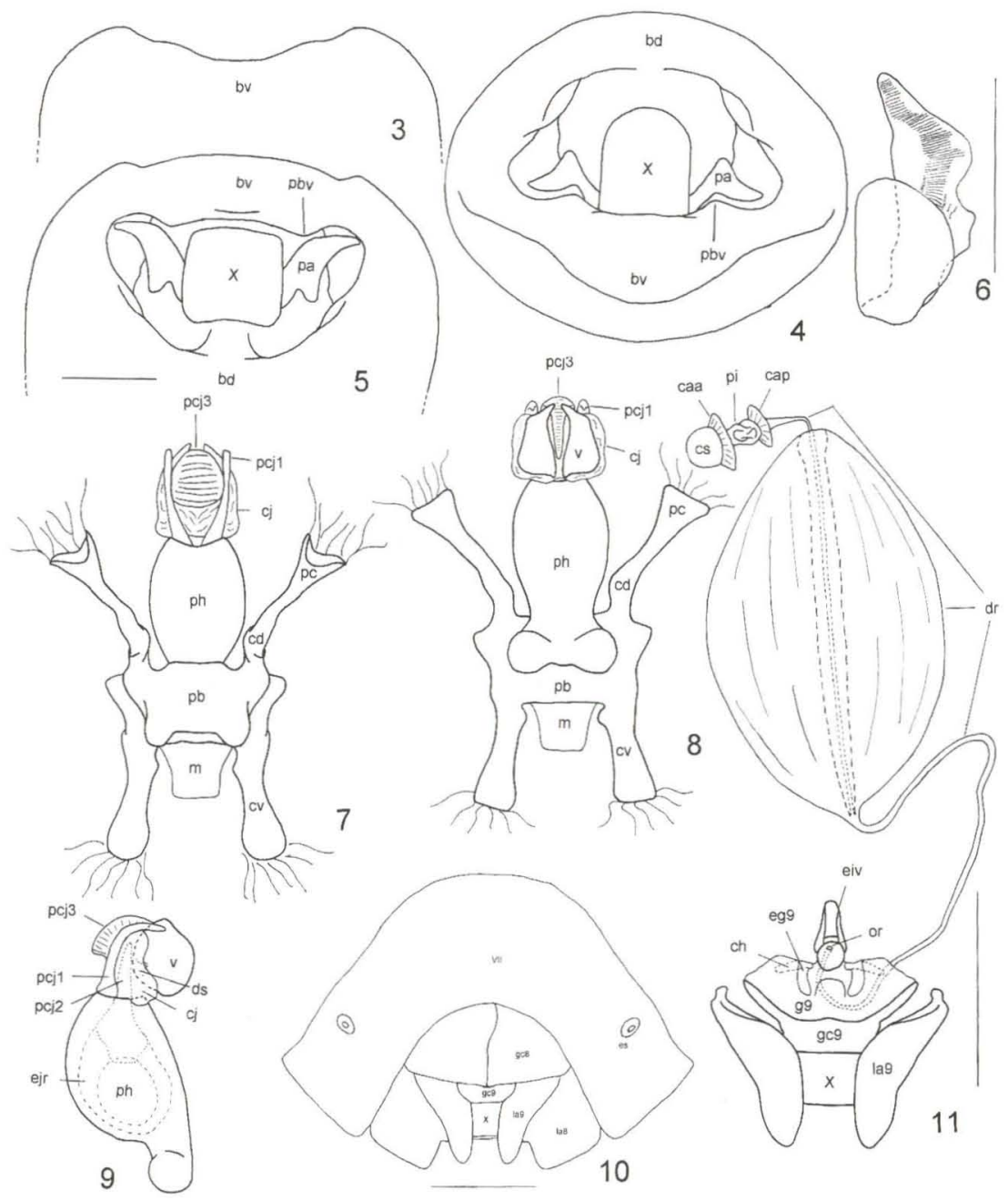

Figs 3-11. (3-9) Parentheca subfurcata macho: (3-5) pigóforo ventral (3), posterior (4), dorsal (5); (6) parâmero direito, lateral externa; (7-9) phallus ventral (7), dorsal (8), lateral (9). (10-11) Fêmea: (10) placas genitais, ventral; (11) laterotergitos, gonocoxitos e gonapófises do nono segmento e vias genitais ectodérmicas, ventral. (bd) Bordo dorsal, (bv) bordo ventral, (caa) crista anular anterior, (cap) crista anular posterior, (cd) conetivo dorsal, (ch) chitinellipsen, (cj) conjuntiva, (cs) capsula seminalis, (cv) conetivo ventral, (dr) ductus receptaculi, (ds) ductus seminis distalis, (eiv) espessamento da intima vaginal, (eg9) espessamentos secundários da gonapófise 9, (ejr) ejaculatory reservoir, (es) espiráculo, (g9) gonapófise 9 , (gc8) gonocoxito 8, (gc9) gonocoxito 9, (la8) laterotergito 8, (la9) laterotergito 9, (m) membramblase, (or) orificium receptaculi, (pa) parâmero, (pb) placa basal, (pbv) processo do bordo ventral, (pc) processus capitati, (pcj1) processo 1 da conjuntiva, (pcj2) processo 2 da conjuntiva, (pcj3) processo 3 da conjuntiva, (ph) phallotheca, (pi) pars intermedialis, (v) vesica, (VII) sétimo urosternito, $(X)$ segmento $X$. Escalas: figuras $3-9=0,5 \mathrm{~mm}$; figuras $10-11=1 \mathrm{~mm}$. 
mentos secundários laterais saculiformes que partem das chitinellipsen (Figs 11,20, eg9, ch), essas de contorno ovalado. Espessamento da íntima vaginal semiesférico em torno do orificium receptaculi e subtriangular anteriormente (Figs 11, 20, eiv, or). Ductus receptaculi (Figs 11,20, dr) de diâmetro reduzido; região anterior à área vesicular mais longa que a região posterior a esta área. Cristas anulares anterior e posterior estreitas e voltadas para a pars intermedialis (Figs 11, 20, caa, cap, pi). Nos 2/3 anteriores da pars intermedialis o ductus receptaculi é enovelado, terço posterior constrito e encoberto pela crista anular posterior. Capsula seminalis destituída de dentes (Figs 11, 20, cs).

Comentários. Parentheca assemelha-se, externamente, a Coenus Dallas, 1851 pelo tamanho e forma geral do corpo. A genitália, especialmente a masculina, sugere uma proximidade a Coenus, Euschistus Dallas, 1851 e Dichelops Spinola, 1837, principalmente devido à presença de processos pares digitiformes na conjuntiva e à forma da vesica. Entretanto, Parentheca é extremamente semelhante ao subgênero Neodichelops Grazia no tocante à presença e forma dos processos do diafragma no pigóforo, estrutura e processos presentes no phallus e enovelamento do ductus receptaculi dentro da pars intermedialis. Na descrição de Dichelops (Neodichelops), GrazIA (1978) define os processos ventrais digitiformes e o processo apical da conjuntiva como pertencentes, respectivamente, à phallotheca $\mathrm{e}$ vesica; com a melhor observação dos processos de Parentheca e comparação com os de Dichelops (Neodichelops) concluímos pela retificação das descrições para esse subgênero, seguindo as adotadas no presente trabalho. São características peculiares de Parentheca a estrutura e dimensão da cabeça, o tamanho diminuto dos olhos, ocelos e peritrema ostiolar e a reduzida abertura da taça genital. Os exemplares examinados apresentam uma evidente braquipteria do segundo par de asas (Fig. 1) e redução na dimensão da membrana do hemiélitro. Este fato poderia limitar a capacidade de vôo nestas espécies. Porém, como o número de exemplares disponível era relativamente pequeno, especialmente de $P$. subfurcata, não é descartada a hipótese da existência de formas plenamente aladas.

\section{Parentheca subfurcata Berg, 1891}

Parentheca subfurcata Berg, 1891: 281. - Kirkaldy, 1909: 90.

Diagnose. Cabeça pouco mais longa que larga, o comprimento excedendo a largura ao nível dos olhos em cerca de $20 \%$. Margens ântero-laterais do pronoto suavemente convexas, íntegras (Fig. 2). Pequena mancha ferrugínea junto ao limite entre os segmentos do conexivo, mais próxima à margem externa.

Redescrição, complementando BERG (1891). Macho: bordo ventral do pigóforo côncavo, levemente sinuoso (Fig. 3). Projeções dorsais do bordo ventral pouco pronunciadas, convexas; área do bordo, entre essas projeções, sub-retilínea (Figs 4, 5). Ângulos póstero-laterais com uma ligeira projeção posterior. Parâmeros com o ramo basal ligeiramente desenvolvido (Figs 4-6). Phallus: placa basal alargada (Figs $7,8)$.

Medidas macho. Cabeça: comprimento 2,5, largura 2,5; artículos antenais: I 0,5 , II 0,9, III 0,7, IV 0,8, V 1,3; pronoto: comprimento 1,8 , largura 4,7; escutelo: comprimento 3,4 , largura 3,2 ; largura abdominal 5,5; comprimento total 9,9. 
Fêmea. Margens posteriores dos gonocoxitos 8 levemente convexas (Fig. 10). Ductus receptaculi antes da área vesicular cerca de oito vezes maior que o ductus após à área vesicular (Fig. 11).

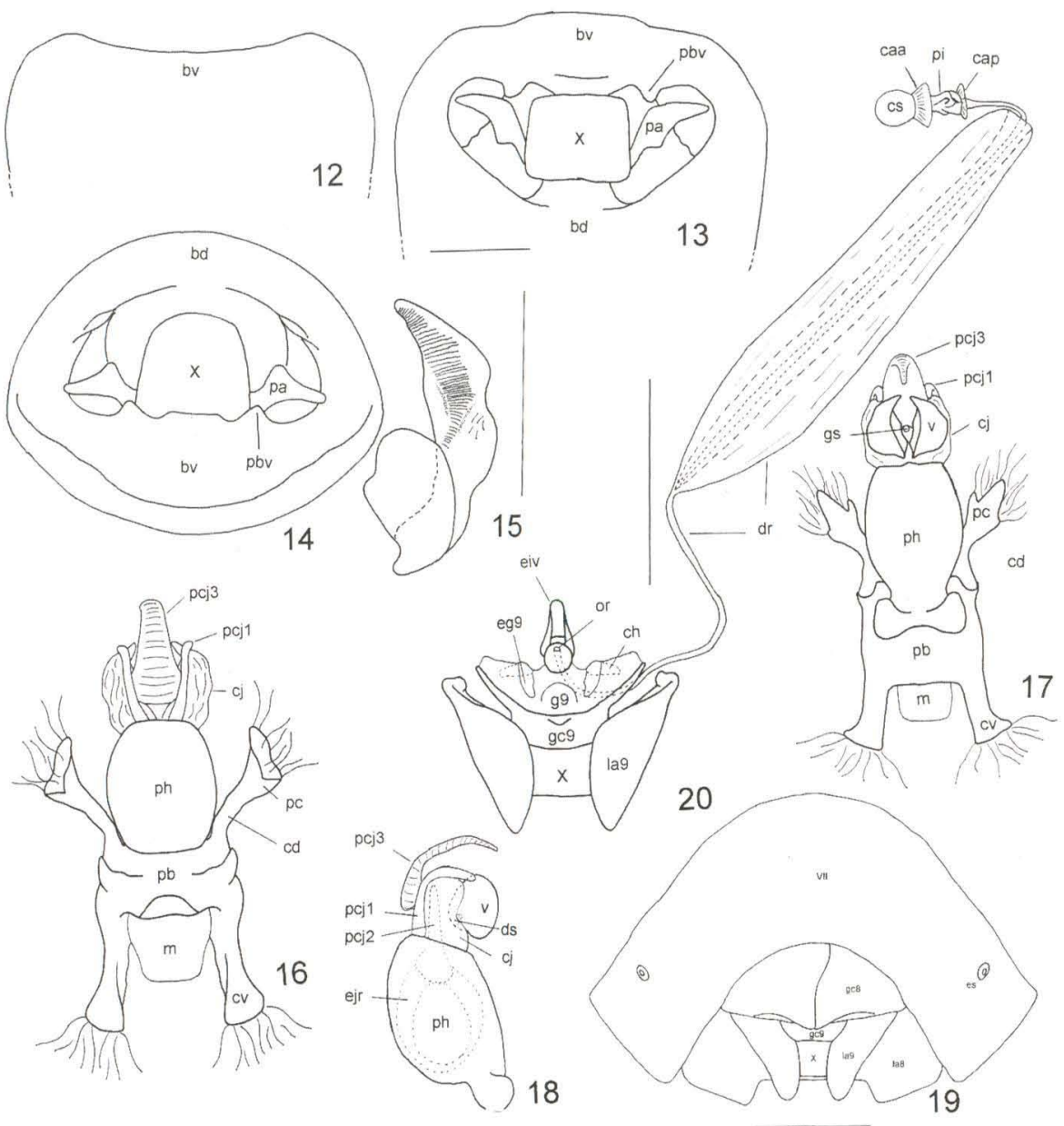

Figs 12-20. Parentheca aeliomorpha macho: (12-14) pigóforo ventral (12), dorsal (13), posterior (14); (15) parâmero direito, lateral externa. (16-18) Phallus ventral (16), dorsal (17), lateral (18). (19-20) Fêmea: (19) placas genitais, ventral; (20) laterotergitos, gonocoxitos e gonapófises do nono segmento e vias genitais ectodérmicas, ventral. (bd) Bordo dorsal, (bv) bordo ventral, (caa) crista anular anterior, (cap) crista anular posterior, (cd) conetivo dorsal, (ch) chitinellipsen, (cj) conjuntiva, (cs) capsula seminalis, (cv) conetivo ventral, (dr) ductus receptaculi, (ds) ductus seminis distalis, (eg9) espessamentos secundários da gonapófise 9, (eiv) espessamento da íntima vaginal, (ejr) ejaculatory reservoir, (es) espiráculo, (g9) gonapófise 9, (gc8) gonocoxito 8, (gc9) gonocoxito 9, (la8) laterotergito 8, (la9) laterotergito 9, (m) membramblase, (or) orificium receptaculi, (pa) parâmero, (pb) placa basal, (pbv) processo do bordo ventral, $(\mathrm{pc})$ processus capitati, ( $\mathrm{pcj} 1$ ) processo 1 da conjuntiva, ( $\mathrm{pcj} 2$ ) processo $2 \mathrm{da}$ conjuntiva, (pcj3) processo 3 da conjuntiva, (ph) phallotheca, (pi) pars intermedialis, (v) vesica, (VII) sétimo urosternito, $(X)$ segmento $X$. Escalas: figuras $12-18=0,5 \mathrm{~mm}$; figuras $19-20=1 \mathrm{~mm}$. 
Medidas fêmea. Cabeça: comprimento 2,69, largura 2,60 (2,52-2,69); artículos antenais: I 0,59 , II 1,01, III $0,97(0,92-1,01)$, IV $0,92(0,84-1,01)$, V falta; pronoto: comprimento 2,27 (2,18-2,35), largura 5,38 (5,21-5,54); escutelo: comprimento $3,78(3,70-3,86)$, largura $3,53(3,36-3,70)$; largura abdominal $6,01(5,80$ $6,22)$; comprimento total 10,96 (10,67-11,26).

Distribuição. Brasil (Minas Gerais), Argentina (Córdoba).

Material examinado. BRASIL, Minas Gerais: Poços de Caldas (Morro do Ferro), macho, 15.XI.1965, J. Becker, O. Roppa, O. Leoncini leg. (CPJB). ARGENTINA, Córdoba, Dep. Calamuchita, "El Sauce", fêmea, XII.1941, M.J. Viana et al. (MACN).

O holótipo de $P$. subfurcata (fêmea) foi examinado, e encontra-se depositado no Museo Argentino de Ciencias Naturales Bernardino Rivadavia (Buenos Aires), com as seguintes etiquetas: (a) Parentheca Berg 1891 (b) Tipo subfurcata Berg (c) et al. antigua (d) Córdoba (e) Typus.

\section{Parentheca aeliomorpha Berg, 1891}

Parentheca aeliomorpha Berg, 1891: 281-282. - Kirkaldy, 1909: 91. - Ruffinelli \& Pirán, 1959: 18. Pirán, 1970: 127.

Diagnose. Cabeça mais longa que larga, o comprimento excede a largura em cerca de $50 \%$. Margens ântero-laterais do pronoto sub-retilíneas, crenuladas em toda a sua extensão (Fig. 1). Face ventral dos fêmures, e as vezes das tíbias, das pernas médias e posteriores com grosseiras manchas ferrugíneas.

Redescrição, complementando BERG (1891). Macho: bordo ventral do pigóforo suave e uniformemente côncavo (Fig. 12), protuberante na região mediana. Projeções dorsais do bordo ventral tuberculares; área do bordo, entre essas projeções, pronunciada em direção ao segmento X (Figs 13, 14). Ângulos póstero-laterais do pigóforo uniformemente arqueados, sem projeções. Ramo basal dos parâmeros pouco desenvolvido (Figs 13-15). Phallus: comprimento e largura da placa basal subiguais (Figs 16, 18).

Medidas macho. Cabeça: comprimento 3,02, largura 2,44 (2,35-2,52); artículos antenais: I 0,67 , II $1,13(1,09-1,18)$, III 0,92 , IV 1,01, V 1,22 (1,18-1,26) pronoto: comprimento 2,02, largura 4,62 (4,54-4,70); escutelo: comprimento 3,61 $(3,53-3,70)$, largura $3,02(2,86-3,19)$; largura abdominal 5,29 (5,21-5,38); comprimento total $10,33(10,25-10,42)$.

Fêmea. Margem posterior dos gonocoxitos 8 sinuosa, ângulos suturais projetados sobre o gonocoxito 9 (Fig. 19). Ductus receptaculi antes da área vesicular cerca de cinco vezes maior que o ductus após a área vesicular (Fig. 20).

Medidas fêmea. Cabeça: comprimento 3,05 (2,35-3,19), largura 2,55 (2,182,69); artículos antenais: I 0,64 (0,50-0,67), II 1,26 (1,09-1,34); III 0,97 (0,95-1,09), IV $1,02(0,76-1,09), \mathrm{V} 1,28$ (1,09-1,34); pronoto: comprimento 2,02 (1,68-2,02), largura 4,98 (4,20-5,21); escutelo: comprimento 3,81 (3,19-4,03), largura 3,28 $(2,69-3,36)$; largura abdominal 5,74 $(5,04-6,05)$; comprimento total $11,16(9,07-$ $11,42)$.

Distribuição. Brasil (Mato Grosso, Goiás, Rio Grande do Sul), Paraguai, Argentina (Chaco, Misiones, Córdoba) e Uruguai. 
Material examinado. Brasil, Mato Grosso: Três Lagoas (Faz. Retiro de Telhas), fêmea, 21.V.1964, Exp. Depto. Zool. leg., "Cerrado"(MZSP); Goiás: Jataí, 2 machos, 4 fêmeas, XII.1963, M. Alvarenga leg. (DZUP). ARGENTINA, Chaco: fềmea, 30.XI.1897, S. Venturi leg., nº 5653 (MACN); Misiones: fêmea, 07.III.1897, S. Venturi leg., n 5653 (MACN); Córdoba: Dep. Calamuchita, "El Sauce", 4 fềmeas, XII.1941, M.J. Viana leg. (MACN); Cruz Alta, fêmea, I.1946, Coll. Duret, Parentheca aeliomorpha Berg, det. A.A. Pirán, cum typus comparavit (MACN).

Foram examinados os síntipos de $P$. aeliomorpha ( 1 macho e 1 fêmea) depositados no Museo de La Plata (La Plata, Argentina), os quais são aqui designados lectótipo e paralectótipo, como segue:

Lectótipo macho, etiquetas: (a) 1392/1 (b) Parentheca aeliomorpha 1891 Berg (c) Misiones (d) Typus (e) Lectotype.

Paralectótipo fêmea, etiquetas: (a) 1392/2 (b) Coll. Rolide (c) Typus (d) Paraguay (e) Paralectotype.

Segundo BERG (1891) um exemplar de $P$. aeliomorpha foi depositado no Museu de Montevideo; infelizmente não foi localizado.

$P$. subfurcata e $P$. aeliomorpha são muito semelhantes, mas podem ser facilmente diferenciadas através da relação comprimento/largura da cabeça, forma das margens ântero-laterais do pronoto, e caracteres da genitália masculina, especialmente do pigóforo, como a forma do bordo ventral e das projeções dorsais do bordo ventral.

\section{REFERÊNCIAS BIBLIOGRÁFICAS}

Berg, C. 1891. Nova Hemiptera Faunarum Argentinae et Uruguayensis. An. Soc. cient. argent. 32: 280-282.

Campos, L.A. \& J. GraziA. 1995. Paratibraca, um novo gênero de Pentatomini (Heteroptera, Pentatomidae). Iheringia, Sér. Zool., Porto Alegre, (79):163-171.

DuPuIs, C. 1955. Les génitalia des Hémiptères-Héteroptères (Génitalia externes des deux sexes, voies ectodermiques femelles). Mém. Mus. Nat. Hist. nat., Paris, A n.s., 6 (4): 183-278.

1970. Heteroptera, p.190-208. In: S.L. TUXEN (Ed.). Taxonomist's Glossary of Genitalia of Insects. Copenhagen, Munksgaard, 359p.

GraZIA, J. 1978. Revisão do gênero Dichelops Spinola, 1837 (Heteroptera, Pentatomidae, Pentatomini). Iheringia, Sér. Zool., Porto Alegre, (53): 3-119.

KirKaldy, G.W. 1909. Catalogue of the Hemiptera (Heteroptera). 1-Cimicidae. Berlin, Felix L. Dames, XL+392p.

PIRÁN, A.A. 1970. Hemiptera Neotropica. XIII. Acta zool. Lilloana 26 (8): 117128.

Rolston, L.H. \& F.J.D. MCDonald. 1984. A conspectus of Pentatomini of the Western Hemisphere. Part 3 (Hemiptera: Pentatomidae). Jour. N.Y. Ent. Soc. 92 (1): 69-86.

RUfFInElli, A. \& A.A. PIRÁN. 1959. Hemipteros Heteropteros del Uruguay. Bol. Fac. Agron. Montevideo 51: 1-18.

Recebido em 13.I.1998; aceito em 06.VI.1999. 